\title{
Nonlinear equations with a generalized fractional Laplacian
}

\section{Igor Kossowski ${ }^{1}$ (D) Bogdan Przeradzki ${ }^{1}$}

Received: 19 December 2019 / Accepted: 2 January 2021 / Published online: 23 January 2021

(c) The Author(s) 2021

\begin{abstract}
We study a generalization of the power of Laplace operator with null Dirichlet conditions by means of the spectral theory and prove several existence results for nonlinear equations with such operators, especially when the problem is resonant. Some regularity results are also obtained.
\end{abstract}

Keywords Fractional derivatives $\cdot$ spectrum of the Laplacian

AMS Subject Classification 35R11 $47 \mathrm{~A} 60 \cdot 47 \mathrm{~J} 05$

\section{Introduction}

Fractional differential operators are modern tools in the modeling of many phenomena of mechanics, electric circuits and material sciences. However, this notion has several meanings starting from fractional derivatives of Riemann-Liouville, Caputo or other kinds, through pseudo-differential and singular integral operators up to definitions given by the Spectral Theory. Some definitions are equivalent though they seem to be completely different, see [16]. They have rather global nature in a sense that functions being their arguments have to be defined on the whole $\mathbb{R}^{k}$. If such operators are applied in equations on an open bounded set $\Omega \subset \mathbb{R}^{k}$ with the null Dirichlet's boundary condition, then the authors set $u=0$ outside $\Omega$. Operators of this kind are generalizations of the Laplace operator and they show some probabilistic motivation (Lévy processes) of models considered [2,16,17,24].

In this paper, we use operator defined by the Dirichlet Laplacian $-\Delta: L^{2}(\Omega) \supset$ $\operatorname{dom}(-\Delta) \rightarrow L^{2}(\Omega)$ by using the Spectral Theorem and functional calculus given in [8]. When $g$ is a real function defined on the spectrum of $-\Delta$, then $g(-\Delta)$ is a certain self-adjoint operator on $L^{2}(\Omega)$. This approach includes positive powers $(-\Delta)^{s}$, but operators defined in this way are different from the ones considered above. The arguments in [23] show that eigenfunctions of global $(-\Delta)^{s}$ differ from the eigenfunctions of the Laplacian which are the same for $g(-\Delta)$ for any function $g$; earlier the eigenvalues of these both operators were compared in [6]. Some existence and regularity results for equations involving spectral fractional Laplacians are given in $[1,4,5,14,21,25]$. It would be interesting to compare results of

Igor Kossowski

igor.kossowski@p.lodz.pl

1 Institute of Mathematics, Lodz University of Technology, ul. Wólczańska 215, 93-005 Łódź, Poland 
this paper with theorems from the above mentioned articles even for fractional Laplacians. However, $[1,4,5,25]$ consider superlinear nonlinearities and our approach admits only ones with linear or sublinear growth (comp. (3.2)). In [21], properties of the fractional Laplacians with Navier and Dirichlet boundary conditions are studied as linear operators on different Sobolev spaces. The closest to our setting is [14], where the fractional Laplacian is replaced by

$$
\sum_{i, j} \alpha_{i} \alpha_{j}(-\Delta)^{\beta_{i}+\beta_{j}}
$$

The operator acts in $L^{2}(\Omega)$ as in our approach and assumptions on parameters give the compactness of the inverse operator. However, the nonlinear term $f$ is potential, since the method used in [14] is variational (here, we use the fixed point and topological degree methods). As in the case of usual Laplace operator, the results obtained by using the variational method need slightly weaker assumptions as obtained by the topological one. We also refer to some applications of fractional Laplacian $[3,18]$ and fractional $p$-Laplacian $[7,11,26,27]$.

There is no reason to restrict to power functions $g(z)=z^{s}$, since engineers using fractional operators rely on experimental arguments: numerical results obtained with fractional powers of differential operators are closer to experimental data. It is possible that some deviations from power functions will give even better results. The paper is organised as follows: in the next section we remind some facts concerning the Dirichlet Laplacian and the Spectral Theory. In Sect. 3, the existence of solutions for $g(-\Delta) u=f(x, u)$ with invertible $g(-\Delta)$ will be obtained. The proof of these theorems are simple. More interesting is the resonant case, in which this operator has a nontrivial kernel. It happens when $g$ vanishes on some eigenvalue of the Laplacian. The existence theorem is then given with conditions similar to these due to Landesman and Lazer [15] and the proof is based on the coincidence degree technique of Mawhin. The last section is devoted to regularity of solutions to such equations. The results obtained there are not satisfactory in general case when any bounds of the sequence of $L^{2}$ and supremum norms of derivatives of every order are not known. In fact, we can use these results for cuboids.

The theorems of this paper are completely analogous to those available for case of usual Dirichlet Laplacians $g(s)=s$. The existence and uniqueness of a solution obtained by the Schauder Fixed Point Theorem or the Banach Contraction Principle (Theorems 1 and 2) are essentially weaker than the known ones in the case of the fractional Laplacians $g(s)=s^{\beta}$, however the novelty of this paper is about the replacement of the power function by an arbitrary one. The main interesting resonant case (Theorem 4) cannot be applied to fractional Laplacians since $s^{\beta}=0$ only for $s=0$ which is not an eigenvalue of the Dirichlet Laplacian.

The authors are very grateful to the Reviewers for their helpful comments, corrections of misprints and improvements of the text. Special acknowledgements are due to the Reviewer 1 who noticed essential error in the proof of Theorem 5 and proposed a solution.

\section{Dirichlet-Laplace operator and its generalizations}

Let $\Omega$ be an open bounded subset of $\mathbb{R}^{k}$ with Lipschitzian boundary and let $-\Delta$ : $L^{2}(\Omega) \supset \operatorname{dom}(-\Delta) \rightarrow L^{2}(\Omega)$ be the weak Dirichlet-Laplacian (compare [14]), i.e. it is Friedrich's extension of the classical Dirichlet-Laplace operator to a self-adjoint operator. If $(-\Delta)_{s}: L^{2}(\Omega) \supset H_{0}^{1}(\Omega) \cap H^{2}(\Omega) \rightarrow L^{2}(\Omega)$ is the strong Dirichlet-Laplace, then 
$H_{0}^{1}(\Omega) \cap H^{2}(\Omega) \subset \operatorname{dom}(-\Delta)$ and $(-\Delta) u=(-\Delta)_{s} u$ for $u \in H_{0}^{1}(\Omega) \cap H^{2}(\Omega)$. Moreover, for $\Omega$ being of class $C^{2}$ or $\Omega$ being cuboid we have $-\Delta=(-\Delta)_{s}$.

The operator $-\Delta$ is self-adjoint and positive, its spectrum $\sigma(-\Delta)$ consists of positive eigenvalues $\lambda_{n}, n \in \mathbb{N}$, with finite multiplicities being a nondecreasing sequence tending to infinity. If we put each eigenvalue as many times in the sequence as its multiplicity and by $e_{n}$ denote a corresponding normalized in $L^{2}$ eigenfunction than the operator can be written as

$$
-\Delta u=\sum_{n=1}^{\infty} \lambda_{n}\left\langle u, e_{n}\right\rangle e_{n} .
$$

The spectral theory [8] provides us, so called, the operational calculus. If $g: \sigma(-\Delta) \rightarrow \mathbb{R}$ is any function then a new operator $g(-\Delta)$ can be defined by the formula

$$
g(-\Delta) u:=\sum_{n=1}^{\infty} g\left(\lambda_{n}\right)\left\langle u, e_{n}\right\rangle e_{n}
$$

with the domain

$$
\operatorname{dom} g(-\Delta):=\left\{u \in L^{2}: \sum_{n=1}^{\infty} g\left(\lambda_{n}\right)^{2}\left|\left\langle u, e_{n}\right\rangle\right|^{2}<\infty\right\} .
$$

In the special case $g(s)=s^{\beta}$ this operator is the fractional Laplacian. Generally, the correspondence $g \mapsto g(-\Delta)$ has the following algebraic properties:

$$
\begin{aligned}
& \left(g_{1}+g_{2}\right)(-\Delta) \supset g_{1}(-\Delta)+g_{2}(-\Delta), \quad(c g)(-\Delta)=c g(-\Delta), \\
& \left(g_{1} \cdot g_{2}\right)(-\Delta) \supset g_{1}(-\Delta) g_{2}(-\Delta),
\end{aligned}
$$

where $g_{1}, g_{2}$ are real functions on the spectrum, $c$ is a real constant and $C \supset B$ means that operator $C$ is an extension of operator $B$.

$g(-\Delta)$ is a self-adjoint operator for any real function $g$, it is positive, if $g$ takes positive values, it is invertible, if $g\left(\lambda_{n}\right) \neq 0$ for any $n$. If $b:=\inf _{n}\left|g\left(\lambda_{n}\right)\right|>0$, then $g(-\Delta)^{-1}$ is defined on the whole $L^{2}(\Omega)$ :

$$
g(-\Delta)^{-1} u=\sum_{n=1}^{\infty} \frac{1}{g\left(\lambda_{n}\right)}\left\langle u, e_{n}\right\rangle e_{n}
$$

and it is bounded. Moreover, if

$$
\lim _{n \rightarrow \infty}\left|g\left(\lambda_{n}\right)\right|=\infty
$$

then this inverse operator is compact. However, if (2.4) holds but $g$ vanishes for a finite set $G:=\left\{n \in \mathbb{N}: g\left(\lambda_{n}\right)=0\right\}$, then the resolvent

$$
R(\lambda, g(-\Delta)):=(g(-\Delta)-\lambda I)^{-1}=\sum_{n=1}^{\infty} \frac{1}{g\left(\lambda_{n}\right)-\lambda}\left\langle u, e_{n}\right\rangle e_{n}
$$

is meromorphic with the pole 0 an it has the form

$$
R(\lambda, g(-\Delta))=\sum_{n \in G} c_{n}(\lambda)\left\langle u, e_{n}\right\rangle e_{n}+R_{0}(\lambda),
$$

where $\lim _{\lambda \rightarrow 0}\left|c_{n}(\lambda)\right|=\infty$ and $R_{0}$ is holomorphic and compact in a neighborhood of 0 . 
We also need some properties of eigenfunctions $e_{n}$. They are of $C^{\infty}$-class in $\Omega$ (even analytic, see [12], Cor. 8.11). It is well-known that the first eigenvalue is simple, hence $\lambda_{1}<\lambda_{2}$, and the corresponding eigenfunction $e_{1}$ is positive in $\Omega$.

In the one dimensional case eigenvalues can be found explicitely. Assume for simplicity $\Omega=(0, \pi),-\Delta u=-u^{\prime \prime}$, then $\lambda_{n}=n^{2}, e_{n}(x)=\sqrt{2 / \pi} \sin (n x)$. In multidimensional case, one can find them for cuboids, some other polytopes or for balls. We write down the eigenvalues and the eigenfunctions in the case $\Omega=(0, \pi)^{k}$ :

$$
n:=\left(n_{1}, \ldots, n_{k}\right), \quad \lambda_{n}=\sum_{j} n_{j}^{2}, \quad e_{n}\left(x_{1}, \ldots, x_{k}\right)=\sqrt{\frac{2^{k}}{\pi^{k}}} \prod_{j} \sin n_{j} x_{j}
$$

(one can find eigenvalues and eigenfunctions for general cuboids, since they can be obtained from $(0, \pi)^{k}$ by using an appropriate homothety, translation and orthogonal mappings) and in the case of 2-dimensional disk $\Omega=\left\{x \in \mathbb{R}^{2}: x_{1}^{2}+x_{2}^{2}<1\right\}$, in polar coordinates $(r, \theta)$ :

$$
\begin{array}{ll}
\lambda_{0, m}^{2}, & e_{0, m}(r, \theta)=\frac{1}{\sqrt{\pi} J_{0}^{\prime}\left(\lambda_{0, m}\right)} J_{0}\left(\lambda_{0, m} r\right), \\
\lambda_{n, m}^{2}, & e_{n, m, 1}(r, \theta)=\frac{1}{\sqrt{\pi} J_{n}^{\prime}\left(\lambda_{n, m}\right)} J_{n}\left(\lambda_{n, m} r\right) \cos n \theta, \\
& e_{n, m, 2}(r, \theta)=\frac{1}{\sqrt{\pi} J_{n}^{\prime}\left(\lambda_{n, m}\right)} J_{n}\left(\lambda_{n, m} r\right) \sin n \theta,
\end{array}
$$

where $\lambda_{n, m}$ stands for $m$-th positive zero of the Bessel function $J_{n}$, for $n=1,2, \ldots$ they are double eigenvalues (comp. [13]).

In a general case $\Omega \subset \mathbb{R}^{k}$ [13], we only know that if $\Omega_{1} \subset \Omega_{2}$, then

$$
\lambda_{n}\left(\Omega_{2}\right) \leq \lambda_{n}\left(\Omega_{1}\right) .
$$

Moreover, there are two positive constants depending on $\Omega$ such that

$$
c(\Omega) n^{\frac{2}{k}} \leq \lambda_{n}(\Omega) \leq d(\Omega) n^{\frac{2}{k}} .
$$

\section{Elliptic equations with generalized fractional Laplacian}

In this section we are interested in the following equations:

$$
g(-\Delta) u=f(x, u),
$$

where $f: \Omega \times \mathbb{R} \rightarrow \mathbb{R}$ is measurable with respect to $x \in \Omega$, continuous w.r.t. $u \in \mathbb{R}$ and

$$
|f(x, u)| \leq a(x)+b|u|, \quad a \in L^{2}(\Omega), b \geq 0 .
$$

The last condition is necessary and suffient for the Nemytskii operator defined by $f$, i.e. $\mathbf{f}(u)(x)=f(x, u(x))$ to act in $L^{2}(\Omega)$ as a continuous mapping and map bounded sets into bounded ones.

Theorem 1 If $g: \sigma(-\Delta) \rightarrow \mathbb{R}$ satisfies (2.4) and

$$
\inf _{n}\left|g\left(\lambda_{n}\right)\right|>b
$$

where $b$ is a constant from (3.2), then equation (3.1) has a solution.

Proof It is easily seen that (3.1) is equivalent to the existence of a fixed point of operator $g(-\Delta)^{-1} \mathbf{f}: L^{2}(\Omega) \rightarrow L^{2}(\Omega)$. Due to (3.2),

$$
\|\mathbf{f}(u)\| \leq\|a\|+b\|u\|,
$$


where $\|\cdot\|$ stands for the $L^{2}$-norm. Since $\left\|g(-\Delta)^{-1}\right\|=\sup _{n}\left|g\left(\lambda_{n}\right)\right|^{-1}<b^{-1}$ by our assumption, hence

$$
\left\|g(-\Delta)^{-1} \mathbf{f}(u)\right\| \leq\left\|g(-\Delta)^{-1}\right\|(\|a\|+b\|u\|) \leq\|u\|
$$

for $\|u\| \leq R$ with sufficiently large $R$. The operator $g(-\Delta)^{-1} \mathbf{f}$ is compact on any bounded set as both mappings of the composition are continuous, $\mathbf{f}$ maps bounded sets into bounded ones and $g(-\Delta)^{-1}$ is compact. The existence of a solution to (3.1) is a consequence of the Schauder Fixed Point Theorem for the ball centered at 0 with radius $R$.

Uniqueness of solutions can be obtained under another assumptions.

Theorem 2 Let $f$ be a Carathéodory function, $f(\cdot, 0) \in L^{2}(\Omega)$ and there exists $L>0$ such that

$$
|f(x, u)-f(x, v)| \leq L|u-v|
$$

for every $u, v \in \mathbb{R}$ and a.e. $x \in \Omega$. If

$$
L<\inf _{n}\left|g\left(\lambda_{n}\right)\right|
$$

then equation (3.1) has a unique solution.

Proof Notice that assumptions on $f$ from this theorem imply (3.2) with $a(\cdot)=f(\cdot, 0)$ and $b=L$. Moreover

$$
\begin{aligned}
& \left\|g(-\Delta)^{-1} \mathbf{f}(u)-g(-\Delta)^{-1} \mathbf{f}(v)\right\| \leq\left\|g(-\Delta)^{-1}\right\|\|\mathbf{f}(u)-\mathbf{f}(v)\| \\
& \leq\left(\inf \left|g\left(\lambda_{n}\right)\right|\right)^{-1} L\|u-v\|
\end{aligned}
$$

and our assumption gives $g(-\Delta)^{-1} \mathbf{f}$ is a contraction on $L^{2}(\Omega)$. The assertion follows from the Banach Contraction Principle.

The above theorems consider equations with invertible linear part $g(-\Delta)$. We can also study resonant equations, when function $g$ vanishes on some eigenvalues. The first result for the Laplace operator was obtained by Landesman and Lazer in 1970 and we generalize it for our operators. The most satisfactory result can be obtained in the case $g\left(\lambda_{1}\right)=0, g$ satisfies condition (2.4) and $\inf _{n \geq 2}\left|g\left(\lambda_{n}\right)\right|>0$.

Lemma 1 Assume $f: \Omega \times \mathbb{R} \rightarrow \mathbb{R}$ is a Carathéodory function satisfying (3.2). If there exist limits

$$
\lim _{u \rightarrow \pm \infty} f(x, u)=: f_{ \pm}(x),
$$

uniform with respect to $x$ and $f_{ \pm} \in L^{2}(\Omega)$, then there is a function $h \in L^{2}(\Omega)$ such that

$$
|f(x, u)| \leq h(x)
$$

for any $(x, u)$.

Proof Due to (3.4) there exists $c>0$ such that

$$
\begin{aligned}
& \left|f(x, u)-f_{+}(x)\right| \leq 1, \quad u>c, \quad \text { a.e. } x \in \Omega, \\
& \left|f(x, u)-f_{-}(x)\right| \leq 1, \quad u<-c, \quad \text { a.e. } x \in \Omega .
\end{aligned}
$$

Then the assertion holds with

$$
h(x)=\max \left\{\left|f_{+}(x)\right|+1,\left|f_{-}(x)\right|+1, a(x)+b c\right\} .
$$


In 1972 Jean Mawhin [19] introduced a topological degree for pairs of maps $(L, N)$, where $L$ was a linear Fredholm map of index 0, $L: X \supset \operatorname{dom} L \rightarrow Z$, and $N: X \rightarrow Z$ which generalized the Leray-Schauder theory for pairs $(I, N)$ with $N$ completely continuous maps. We follow two later monographs of this theory [10,20]. Let $X$ and $Z$ be Banach spaces, $L: X \supset Y \rightarrow Z$ be a linear operator such that $\operatorname{dim} \operatorname{ker} L=\operatorname{codim} \operatorname{im} L>0$. (Such operators are called Fredholm with index 0. More generally, Fredholm operators have finite dimensional kernels, closed images with finite dimensional $Z / \operatorname{im} L$. The index of the operator is the difference of these dimensions.) Let $N: X \rightarrow Z$ be continuous (and nonlinear). Let $P$ be a linear projector on $\operatorname{ker} L$ and $Q$ a linear projector in $Z$ along im $L$. Denote by $K_{P}$ the inverse of

$$
L \mid(\operatorname{ker} P \cap Y): \operatorname{ker} P \cap Y \rightarrow \operatorname{im} L .
$$

The operator $N$ is called $L$-compact if $Q N$ and $K_{P}(I-Q) N$ are completely continuous (usually $K_{P}$ is completely continuous and $N$ maps bounded sets into bounded ones).

Theorem 3 (Mawhin's Continuation Theorem [10]) Let $U \subset X$ be open and bounded, L be a Fredholm operator of index $0, N$ be L-compact operator and $J$ be an arbitrary isomorphism of im $Q$ onto $\operatorname{ker} L$. Suppose that equations

$$
L x=\mu N(x)
$$

have no solutions on $\partial U \cap Y$ for any $\mu \in(0,1]$ and the Brouwer degree

$$
\operatorname{deg}(J Q N \mid \operatorname{ker} L, U \cap \operatorname{ker} L, 0) \neq 0 .
$$

Then equation $L x=N(x)$ has a solution in $\bar{U}$. The Brouwer degree in the main assumption is often called coincidence degree of $L$ and $N$.

Here, $X=Z=L^{2}(\Omega), L=g(-\Delta), N=\mathbf{f}, \operatorname{ker} L=\operatorname{Lin}\left\{e_{1}\right\}, \operatorname{im} L=\operatorname{ker} L^{\perp}, Z / \operatorname{im} L$ can be identified with ker $L, P, Q$ are orthogonal projectors, $J=I, K_{P}=\left.g(-\Delta)\right|_{\operatorname{ker} L^{\perp}} ^{-1}$ : $\operatorname{im} L \rightarrow \operatorname{ker} L^{\perp}$.

Theorem 4 Suppose $g\left(\lambda_{1}\right)=0$, $g$ satisfies condition (2.4) and $\inf _{n \geq 2}\left|g\left(\lambda_{n}\right)\right|>0$ and $f$ is a Carathéodory function satisfying (3.2) and (3.4). If the following integrals have opposite signs:

$$
\int_{\Omega} f_{+} \cdot e_{1}, \quad \int_{\Omega} f_{-} \cdot e_{1},
$$

then (3.1) has a solution.

Proof It is obvious that $g(-\Delta)$ has index 0 with kernel and cokernel being one-dimensional spaces spanned by $e_{1}$. Using the notation of Mawhin's Continuation Theorem

$$
K_{P}(I-Q) N(u)=\sum_{n \geq 2} \frac{1}{g\left(\lambda_{n}\right)}\left\langle\mathbf{f}(u), e_{n}\right\rangle e_{n},
$$

where $\|\mathbf{f}(u)\| \leq\|h\|$ for any $u \in L^{2}(\Omega)$ by the lemma. This operator is then compact due to

$$
\frac{1}{g\left(\lambda_{n}\right)} \rightarrow 0, \quad n \rightarrow \infty .
$$

We shall show that equations $g(-\Delta) u=\mu \mathbf{f}(u)$ have no solution for $\mu \in(0,1]$ with the norm sufficiently large. Suppose the converse: there are $\mu_{j} \in(0,1]$ and $u_{j} \in L^{2}$, such that

$$
g(-\Delta) u_{j}=\mu_{j} \mathbf{f}(u), \quad \lim _{j \rightarrow \infty}\left\|u_{j}\right\|=\infty .
$$


Denoting by $\tilde{u}$ the orthogonal projection of $u$ on ker $L^{\perp}$, we can write $u_{j}=\tilde{u}_{j}+d_{j} e_{1}$. Then we have

$$
\begin{aligned}
& \left\|\tilde{u}_{j}\right\|^{2}=\sum_{n \geq 2} \frac{\mu_{j}^{2}}{g\left(\lambda_{n}\right)^{2}}\left\langle\mathbf{f}\left(u_{j}\right), e_{n}\right\rangle^{2} \leq\left\|\left(\left.g(-\Delta)\right|_{e_{1}^{\perp}}\right)^{-1} \mathbf{f}\left(u_{j}\right)\right\|^{2} \\
& \leq \frac{1}{\inf _{n \geq 2} g\left(\lambda_{n}\right)^{2}}\|h\|^{2}=: M,
\end{aligned}
$$

hence the norms of $\tilde{u}_{j}$ are uniformly bounded so the sequence $\left|d_{j}\right|$ tends to the infinity. We shall show that both possibilities: there is a subsequence $d_{j} \rightarrow+\infty$ (resp. $d_{j} \rightarrow-\infty$ ) lead to a contradiction in the same way. Take the first option into account and let $\left(d_{j}\right)$ stands for the corresponding subsequence.

Denote $A_{j}:=\left\{x \in \Omega:\left|\tilde{u}_{j}(x)\right|>\frac{1}{2} d_{j} e_{1}(x)\right\}$. Since

$$
\left\|\tilde{u}_{j}\right\|^{2} \geq \int_{A_{j}}\left|\tilde{u}_{j}\right|^{2} \geq \frac{d_{j}^{2}}{4} \int_{A_{j}} e_{1}^{2},
$$

the sequence of integrals of $e_{1}^{2}$ over $A_{j}$ tends to 0 . Take any $\varepsilon>0$. Hence

$$
\mu_{k}\left\{x \in A_{j}: e_{1}(x)>\varepsilon\right\} \varepsilon^{2} \leq \int_{A_{j}} e_{1}^{2} \rightarrow 0
$$

where $\mu_{k}$ denotes the $k$-dimensional Lebesgue measure, thus $\mu_{k}\left(A_{j}\right) \rightarrow 0$, since $\varepsilon>0$ is arbitrary. Passing to a subsequence, we can assume

$$
\sum_{j=1}^{\infty} \mu_{k}\left(A_{j}\right)<\infty
$$

Due to the Borel-Cantelli Lemma

$$
\mu_{k}\left(\bigcap_{i} \bigcup_{j \geq i} A_{j}\right)=0
$$

and

$$
\Omega \backslash \bigcap_{i} \bigcup_{j \geq i} A_{j}=\bigcup_{i} \bigcap_{j \geq i}\left(\Omega \backslash A_{j}\right) \subset \bigcup_{i} \bigcap_{j \geq i}\left\{x: \tilde{u}_{j}(x)+d_{j} e_{1}(x)>\frac{1}{2} d_{j} e_{1}(x)\right\} .
$$

On the other hand $e_{1}$ is positive on $\Omega$, thus $\frac{1}{2} d_{j} e_{1}(x) \rightarrow+\infty$ as $j \rightarrow \infty$ and, therefore $\tilde{u}_{j}(x)+d_{j} e_{1}(x) \rightarrow+\infty$ a.e. and

$$
\lim _{j \rightarrow \infty} f\left(x, \tilde{u}_{j}(x)+d_{j} e_{1}(x)\right)=f_{+}(x), \text { a.e. } x .
$$

Let us multiply both sides of equation (3.6) by $e_{1}$ and integrate it over $\Omega$ and divide by $\mu_{j}$. The left-hand integral vanishes since the image of $g(-\Delta)$ is orthogonal to $e_{1}$. The right-hand side tends to $\int_{\Omega} f_{+} e_{1}$ by Lebesgue's Dominated Convergence Theorem, so it is nonzero for sufficiently large $j$. We have a contradiction. The second option gives the same with $f_{-}$ instead of $f_{+}$.

Thus, we can take $R>0$ so large that all solutions of $g(-\Delta) u=\mu \mathbf{f}(u)$ sit in the open ball $B(0, R)$ in $L^{2}(\Omega)$. We should calculate the Brouwer degree of the mapping

$$
\mathbb{R} \ni d \mapsto \int_{\Omega} \mathbf{f}\left(d e_{1}\right) \cdot e_{1}
$$


on the interval $(-R, R)$. But these mappings take values with the same signs as (3.5) on both ends of this interval. Therefore this degree is +1 or -1 in the cases

$$
\int_{\Omega} f_{+} \cdot e_{1}>0>\int_{\Omega} f_{-} \cdot e_{1}, \quad \int_{\Omega} f_{+} \cdot e_{1}<0<\int_{\Omega} f_{-} \cdot e_{1},
$$

respectively. By Mawhin's Continuation Theorem we get the assertion.

Similar theorem can be obtained replacing $\lambda_{1}$ by any simple eigenvalue $\lambda_{m}$. Since the corresponding eigenfunction $e_{m}$ change signs, the main assumption (3.5) should be changed by: the integrals

$$
\begin{aligned}
& \int_{\left\{x \in \Omega: e_{m}(x)>0\right\}} f_{+} e_{m}+\int_{\left\{x \in \Omega: e_{m}(x)<0\right\}} f_{-} e_{m}, \\
& \int_{\left\{x \in \Omega: e_{m}(x)<0\right\}} f_{+} e_{m}+\int_{\left\{x \in \Omega: e_{m}(x)>0\right\}} f_{-} e_{m}
\end{aligned}
$$

have opposite signs.

One can also replace the limits in definitions $f_{ \pm}$by limes inferior and superior as in [9]. Namely, if

$$
\int_{\Omega} \liminf _{u \rightarrow+\infty} f(\cdot, u) e_{1}>0>\int_{\Omega} \limsup _{u \rightarrow-\infty} f(\cdot, u) e_{1}
$$

or

$$
\int_{\Omega} \liminf _{u \rightarrow-\infty} f(\cdot, u) e_{1}>0>\int_{\Omega} \limsup _{u \rightarrow+\infty} f(\cdot, u) e_{1}
$$

and the limit superior and limit inferior exist uniformly with respect to $x$, then a solution exists.

\section{Some regularity results}

There could be obtained two kinds of regularity theorems. Both of them rely on some boundedness properties of the sequence of eigenfunctions $e_{n}$ which can be checked in some cases. However, in general, they are difficult to verify. Assume that $u$ is a solution of (3.1) where function $g$ can vanish for a finite number of $\lambda_{n}$ and $f$ satisfies (3.2). We consider the question when a solution $u$ belongs to the Sobolev space $H^{p}(\Omega)$. In this section, we assume that all eigenfunctions are sufficiently smooth up to the boundary.

Let $\nabla^{p} u$ denote the $p+1$-tensor $D^{\alpha} u$, where $\alpha=\left(\alpha_{1}, \ldots \alpha_{k}\right) \in \mathbb{N}^{k}$, with entries

$$
\frac{\partial^{|\alpha|} u}{\partial x_{1}^{\alpha_{1}} \ldots \partial x_{k}^{\alpha_{k}}}, \quad|\alpha|=\sum_{j} \alpha_{j}=p
$$

and

$$
\left\|\nabla^{p} u\right\|^{2}:=\int_{\Omega} \sum_{|\alpha|=p}\left|D^{\alpha} u\right|^{2}
$$

is given by corresponding scalar products. We need an estimate:

$$
\left\|\nabla^{p} e_{n}\right\|^{2} \leq C \lambda_{n}^{p}, \quad p \in \mathbb{N}
$$


for a positive constant $C$ depending on $\Omega$ only. In particular, it means that $e_{n} \in H^{p}(\Omega)$ for $n \in \mathbb{N}$. The second assumption is the orthogonality of vectors $\nabla^{p} e_{n}$ and $\nabla^{p} e_{m}$ for $n \neq m$ and any $p$ :

$$
\left\langle\nabla^{p} e_{n}, \nabla^{p} e_{m}\right\rangle=0, \quad n \neq m, \quad p \in \mathbb{N} .
$$

If $p=1$, we have only

$$
\left\|\nabla e_{n}\right\|^{2}=\lambda_{n}, \quad\left\langle\nabla e_{n}, \nabla e_{m}\right\rangle=0, \text { for } n \neq m
$$

obtained with using integration by parts. For $p>1$, the question is more complicated.

Lemma 2 If $\partial \Omega$ is piecewise smooth,

$$
\begin{aligned}
& \int_{\partial \Omega}\left\langle\frac{\partial}{\partial v} \nabla^{p-1} e_{n}, \nabla^{p-1} e_{n}\right\rangle_{e} \leq 0, \\
& \int_{\partial \Omega}\left\langle\frac{\partial}{\partial v} \nabla^{p-1} e_{n}, \nabla^{p-1} e_{m}\right\rangle_{e}=0
\end{aligned}
$$

for any $p>1, n \in \mathbb{N}$ and $m \neq n$, then (4.1) holds with $C=1$ and (4.2) is satisfied too, where $\langle\cdot, \cdot\rangle_{e}$ stands for the scalar product in Euclidean spaces.

Proof We can integrate by parts:

$$
\begin{aligned}
& \int_{\Omega}\left\langle\nabla^{p} e_{n}, \nabla^{p} e_{m}\right\rangle_{e}=\sum_{i_{1}, \ldots, i_{p}} \int_{\Omega} \partial_{x_{i_{1}}, \ldots, x_{i_{p}}} e_{n} \cdot \partial_{x_{i_{1}}, \ldots, x_{i p}} e_{m} \\
& =\sum_{i_{1}, \ldots, i_{p-1}}\left(\int_{\Omega} \partial_{i_{1}, \ldots, i_{p-1}}\left(-\Delta e_{n}\right) \cdot \partial_{i_{1}, \ldots, i_{p-1}}\left(e_{m}\right)+\int_{\partial \Omega} \frac{\partial}{\partial v} \partial_{i_{1}, \ldots, i_{p-1}}\left(e_{n}\right) \cdot \partial_{i_{1}, \ldots, i_{p-1}}\left(e_{m}\right)\right) .
\end{aligned}
$$

The first summands equals 0 for $m \neq n$ or equals $\lambda_{n}\left\|\nabla^{p-1} e_{n}\right\|$ for $m=n$ by induction and the second ones are given by the assumptions of this lemma.

It is known that if domain is $C^{p, \alpha}$, then $e_{n} \in C^{p, \alpha}(\bar{\Omega})$ (compare Theorem 6.19 in [12]), hence $\left\|\nabla^{p} e_{n}\right\|$ is finite. In general, $\left\|\nabla^{p} e_{n}\right\|$ need not to be finite if $p \geq 2$. We will not focus on the question of the finiteness of this norm, since (4.1), (4.2) require much more than this problem. We have written eigenvalues and eigenfunctions for normalized cuboids (2.5). The eigenfunctions are from $C^{\infty}(\bar{\Omega})$. One can easily check that (4.1) holds for cuboids with $C=1$ (both sides are then equal). Simple calculations show that (4.2) is also true for these normalized eigenfunctions. General cuboids are images of the normalized one by an appropriate affine mapping being a composition of an orthogonal map, a homothety and a translation. These maps change eigenvalues multiplying them by $c^{k}$, where $c$ is the ratio of the homothety. The eigenfunctions are the composition of the normalized one with the above affine map. Thus, $e_{n} \in C^{\infty}(\bar{\Omega}), n \in \mathbb{N}$, and relations (4.1), (4.2) hold for a general cuboid.

We know also the explicite form of eigenfunctions for a disk. However, in this case the problem is with the orthogonality of higher derivatives of different eigenfunctions and we are not able to check the needed conditions.

Finally, we want to emphasize that, to the best of our knowledge, conditions (4.1) and (4.2) do not follow from known properties of eigenfunctions for general domains. Only the explicit form of eigenfunctions can be used to obtain estimates (4.1) and (4.2). 
Theorem 5 If $u$ is a solution of (3.1), (4.1), (4.2) are satisfied, $g$ vanishes on $\lambda_{n}$ with $n \in G$, $\operatorname{card}(G)<\infty$, and sequence

$$
\frac{\lambda_{n}^{p}}{\left|g\left(\lambda_{n}\right)\right|^{2}}, \quad n \notin G,
$$

is bounded, then $u \in H^{p}(\Omega)$.

Proof Let $\tilde{u}$ denotes the orthogonal projection of $u$ on $\operatorname{ker} g(-\Delta)^{\perp}$ and $u=\tilde{u}+\sum_{n \in G} d_{n} e_{n}$. Since eigenfunctions $e_{n}, n \notin G$, belong to $H^{p}(\Omega)$, we should only check if $\tilde{u} \in H^{p}(\Omega)$. But

$$
\tilde{u}=\sum_{n \notin G} \frac{1}{g\left(\lambda_{n}\right)}\left\langle\mathbf{f}(u), e_{n}\right\rangle e_{n}
$$

where all entries of the series sit in $H^{p}(\Omega)$. By (4.2),

$$
\left\|\nabla^{m} \tilde{u}\right\|^{2}=\sum_{n \notin G} \frac{1}{\left|g\left(\lambda_{n}\right)\right|^{2}}\left|\left\langle\mathbf{f}(u), e_{n}\right\rangle\right|^{2}\left\|\nabla^{m} e_{n}\right\|^{2}
$$

for $m \leq p$, we have from (4.1) and (4.3) that, for $m \leq p$,

$$
\left\|\nabla^{m} \tilde{u}\right\|^{2}=\sum_{n \notin G} \frac{\left\|\nabla^{m} e_{n}\right\|^{2}}{\left|g\left(\lambda_{n}\right)\right|^{2}}\left|\left\langle\mathbf{f}(u), e_{n}\right\rangle\right|^{2} \leq C\|\mathbf{f}(u)\|^{2},
$$

thus we are done.

Remark If function $g$ is nondecreasing then one can use estimates

$$
c_{1} n^{2 / k} \leq \lambda_{n} \leq c_{2} n^{2 / k}
$$

since $\Omega$ contains a cube and it is a subset of another cube. Thus the assumptions guaranteeing regularity of both kinds do not require an explicit knowledge of eigenvalues but estimates on the growth of norms $\nabla^{p} e_{n}$ are still needed. (4.3) is then equivalent to the boundedness of the sequence

$$
\frac{n^{p / k}}{\left|g\left(n^{2 / k}\right)\right|}, \quad n \notin G .
$$

Corollary 1 Assume (4.1) and (4.2). For the fractional Laplacian $(-\Delta)^{\beta}$, condition $p \leq$ $2 \beta+k$, guarantees $u \in H^{p}(\Omega)$.

The second kind of solution regularity is $u \in C^{p}(\Omega)$. It needs some information about the sequence of supremum norms of $\nabla^{p} e_{n}, p=0,1,2, \ldots$ :

$$
\left\|\nabla^{p} e_{n}\right\|_{\infty}:=\max _{|\alpha| \leq p} \sup _{x \in \Omega}\left|D^{\alpha} e_{n}(x)\right| .
$$

These numbers can be calculated for the case of cuboids and disks directly and the following result can be applied for such simple domains:

Theorem 6 If $u$ is a solution of (3.1), where $g$ vanishes only on $\lambda_{n}$ with $n$ from a finite set $G$ and $f$ is a Carathéodory function satisfying (3.2), then $u \in C^{p}(\Omega)$ provided there exists $C>0$ such that

$$
\left\|\nabla^{p} e_{n}\right\|_{\infty} \leq C \lambda_{n}^{p / 2}
$$


and the series

$$
\sum_{n \notin G} \frac{\lambda_{n}^{p / 2}}{\left|g\left(\lambda_{n}\right)\right|}<\infty
$$

The proof is obvious since all partial derivatives of $\tilde{u}$ up to the order $p$ are sums of uniformly convergent series of continuous functions - multiples of $D^{\alpha} e_{n}$. The crucial assumption (4.4) holds for cubes in any dimension.

Corollary 2 If $g(s)=s^{\beta}$, then $u \in C^{p}$ for $p<2 \beta-k$.

\section{Examples}

Let $\Omega:=(0, \pi) \subset \mathbb{R},-\Delta u=-u^{\prime \prime}$, then $\lambda_{n}=n^{2}, n \in \mathbb{N}$. Consider a fractional power: $g(s):=s^{\beta}$, where $\beta>0$. Then inf $\left|g\left(\lambda_{n}\right)\right|=1$ and we can use Theorem 1 for the following nonlinearities:

- $f(x, u)=h_{1}(x)|u|^{\alpha}+h_{2}(x)$, where $\alpha \in(0,1), h_{1} \in L^{\infty}, h_{2} \in L^{2}$;

- $f(x, u)=h_{1}(x) u+h_{2}(x)$, where $h_{1} \in L^{\infty},\left\|h_{1}\right\|_{\infty}<1, h_{2} \in L^{2}$;

- $f(x, u)=h_{1}(x) u \arctan u+h_{2}(x)$, where $h_{1} \in L^{\infty},\left\|h_{1}\right\|_{\infty}<2 / \pi, h_{2} \in L^{2}$.

The function $f$ given by the last two formulas satisfy assumptions of Theorem 2, thus we have exactly one solution in these cases.

Now, we consider the same problem with $g(s)=s$ and $f(x, u)=u$, then all assumptions of Theorem 1 will be satisfied except $b<\inf _{n} g\left(\lambda_{n}\right)$ - here we have the equality instead the sharp inequality. The same statement is true for Theorem 2 and the assumption $L<$ $\inf _{n} g\left(\lambda_{n}\right)$. However the problem has an obvious solution $u(x)=\sin x$. If we change the nonlinear term and put $f(x, u)=u-1$, then the assumptions of both Theorem 1 and 2 are satisfied except the sharp inequalities but the problem has no solution.

Theorem 4 can be applied to functions $g(s)=s^{\beta}-1$, where $\beta>0$. Then for

$$
f(x, u)=h_{1}(x) \arctan u+h_{2}(x),
$$

where $h_{1} \in L^{\infty}(0, \pi), h_{2} \in L^{2}(0, \pi)$, condition (3.5) has the following form:

$$
\int_{0}^{\pi}\left(h_{2}(x)+\frac{\pi}{2} h_{1}(x)\right) \sin x d x>0>\int_{0}^{\pi}\left(h_{2}(x)-\frac{\pi}{2} h_{1}(x)\right) \sin x d x .
$$

Another example show that the existence of solutions for a resonant case can be obtained also for unbounded nonlinearities $f$ with infinite limits $f_{ \pm}$as $u \rightarrow \pm \infty$ but satisfying (3.5). For usual Laplacian, such a generalization of Theorem 4 is known. Take $\Omega=(0, \pi)$, $g(s)=\sqrt{s}-1, f(x, u)=u+\sqrt{2 / \pi} \sin x$. In this case $f_{ \pm}(x)= \pm \infty$ and (3.5) holds with the integrals $\pm \infty$. Then $u=-e_{1}$ is a solution.

Open Access This article is licensed under a Creative Commons Attribution 4.0 International License, which permits use, sharing, adaptation, distribution and reproduction in any medium or format, as long as you give appropriate credit to the original author(s) and the source, provide a link to the Creative Commons licence, and indicate if changes were made. The images or other third party material in this article are included in the article's Creative Commons licence, unless indicated otherwise in a credit line to the material. If material is not included in the article's Creative Commons licence and your intended use is not permitted by statutory regulation or exceeds the permitted use, you will need to obtain permission directly from the copyright holder. To view a copy of this licence, visit http://creativecommons.org/licenses/by/4.0/. 


\section{References}

1. Barrios, B., Colorado, E., de Pablo, A., Sánchez, U.: On some critical problems for the fractional Laplacian operator. J. Diff. Equ. 252, 6133-6162 (2012)

2. Molica Bisci, G., Radulescu, V., Servadei, R.: Variational Methods for fractional Boundary Problems. Cambridge Univ. Press, Cambridge (2016)

3. Bucur, C., Valdinoci, E.: Nonlocal diffusion and applications, Lecture Notes of the Unione Math. Italiana, 20, Springer, Bologna (2016)

4. Cabre, X., Tan, J.: Positive solutions of nonlinear problems involving the square root of the Laplacian. Adv. Math. 224, 2052-2093 (2010)

5. Capella, A., Dávila, J., Dupaigne, L., Sire, Y.: Regularity of Radial Extremal Solutions for Some NonLocal Semilinear Equations. Commun. Partial Differ. Equ. 36, 1353-1384 (2011)

6. Chen, Z.-Q., Song, R.: Two-sided eigenvalue estimates for subordinate processes in domains. J. Funct. Anal. 226, 90-113 (2005)

7. Chen, J., Cheng, B., Tang, X.: New existence of multiple solutions for nonhomogeneous SchrödingerKirchhoff problems involving the fractional p-Laplacian with sign-changing potential. Rev. R. Acad. Cienc. Exactas F1s. Nat. Ser. A Mat. RACSAM 112, 153-176 (2018)

8. Conway, J.B.: A Course in Functional Analysis. Springer-Verlag, New York (1990)

9. Drábek, P.: On the resonance problem with nonlinearity which has arbitrary linear growth. J. Math. Anal. Appl. 127, 435-442 (1987)

10. Gaines, R.E., Mawhin, J.: Coincidence Degree and Nonlinear Differential Equations. Lecture Notes in Math, vol. 568. Springer-Verlag, Berlin, Heidelberg, New York (1977)

11. Ge, B., Sun, L.-L., Cui, Y.-X., Ferrara, M., Zhao, T.-T.: Infinitely many solutions for a class of elliptic problems involving the fractional Laplacian. Rev. R. Acad. Cienc. Exactas Fis. Nat. Ser. A Mat. RACSAM 113, 657-673 (2019)

12. Gilbarg, D., Trudinger, N.S.: Elliptic Partial Differential Equations of Second Order. Springer, Berlin, Heidelberg, New York (2001)

13. Henrot, A.: Extremum Problems for Eigenvalues of Elliptic Operators. Birkhäuser, Basel, Boston, Berlin (2006)

14. Idczak, D.: A bipolynomial fractional Dirichlet-Laplace problem. Electron. J. Differ. Equ. 2019(59), 1-17 (2019)

15. Landesman, E.M., Lazer, A.C.: Nonlinear perturbations of linear elliptic boundary value problems at resonance. J. Math. Mech. 19, 609-623 (1970)

16. Kwaśnicki, M.: Ten equivalent definitions of the fractional Laplace operator. Fract. Calc. Appl. Anal. 20, 7-51 (2017)

17. Kwaśnicki, M.: Fractional Laplace operator and its properties, Handbook of fractional calculus with applications. Vol. 1, 159-193, De Gruyter, Berlin, (2019)

18. de la Llave, R., Valdinoci, E.: A generalization of Aubry-Mather theory to partial differential equations and pseudodifferential equations. Ann. Inst. H. Poincaré Anal. Nonl. 26(4), 1309-1344 (2009)

19. Mawhin, J.: Equivalence theorems for nonlinear operator equations and coincidence degree theory for some mappings in locally convex topological vector spaces. J. Differ. Equ. 12, 610-636 (1972)

20. Mawhin, J.: Topological Degree Methods in Nonlinear Boundary Value Problems, CBMS Reg. Conf. in Math., No 40, American Math. Soc., Providence, RI, (1979)

21. Musina, R., Nazarov, A.I.: On fractional Laplacians. Commun. Partial Differ. Equ. 39(9), 1780-1790 (2014)

22. Przeradzki, B.: A new continuation method for the study of nonlinear equations at resonance. J. Math. Anal. Appl. 180, 553-565 (1993)

23. Servadei, R., Valdinoci, E.: On the spectrum of different two fractional operators. Proc. R. Soc. Edinburgh Sect. A 144, 831-855 (2014)

24. Servadei, R.: A critical fractional Laplace equation in the resonant case. Topol. Methods Nonlinear Anal. 43, 251-267 (2014)

25. Tan, J.: The Brezis-Nirenberg type problem involving the square root of the Laplacian. Calc. Var. 42, 21-41 (2011)

26. Zhang, Y., Tang, X., Zhang, J.: Existence of infinitely many solutions for fractional p-Laplacian Schrödinger-Kirchhoff type equations with sign-changing potential. Rev. R. Acad. Cienc. Exactas Fis. Nat. Ser. A Mat. RACSAM 113, 569-586 (2019)

27. Zhang, Y., Tang, X., Zhang, J.: Existence and multiplicity of solutions for Kirchhoff type equations involving fractional p-Laplacian without compact condition. Rev. R. Acad. Cienc. Exactas Fis. Nat. Ser. A Mat. RACSAM 113, 3147-3167 (2019) 
Publisher's Note Springer Nature remains neutral with regard to jurisdictional claims in published maps and institutional affiliations. 